\title{
GW23-e0300 E64D DETERIORATES POST-MYOCARDIAL INFARCTION LEFT VENTRICULAR REMODELLING BY INHIBITING CATHEPSIN S-MEDIATED FIBROBLAST TRANS- DIFFERENTIATION
}

doi:10.1136/heartjnl-2012-302920q.2

Han Chen, Jian-An Wang. Department of Cardiology, the Second Affiliated Hospital, College of Medicine, Zhejiang University, Hangzhou, China

Objectives Extracellular matrix (ECM) turnover is a major process of left ventricular (LV) remodelling following myocardial infarction (MI). Cysteinyl cathepsins participate in ECM catabolism in human arterial diseases, but their functions in cardiac remodelling remains unknown.

Methods Mouse MI model was induced by left anterior descending (LAD) artery ligation. Both infarct and remote myocardium from post-MI 1, 2, 3, 7 and 28 days were collected to evaluate mRNA expressions and activities of different cysteinyl cathepsins comparing to sham operated ones. To further investigate the role of cathepsins in post-MI LV remodelling process, a non-selective cysteinyl cathepsin inhibitor E64d was administrated within the first 7 days of post-MI. Cardiac functions were analysed by echocardiography at baseline, 7 and 28 days post-MI. Mice were sacrifice at 7 and 28 days post MI for further studies.

Results Cats expression and activity were increased in infarcted mouse myocardium. E64d administration deteriorated cardiac functions at 7 and 28 days post-MI, although did not change significantly infarct size. This cathepsin inhibitor increased post-MI inflammatory cell infiltration and cytokine expression, altered collagen type-I and type-III deposition, and suppressed the expressions of myofibroblast trans-differentiation-essential protein fibronectin extra domain A (ED-A) and myofibroblast marker a-smooth muscle actin (a-SMA), but did not affect myocardium apoptosis or angiogenesis. Further mechanistic studies demonstrated that inhibition or deficiency of CatS reduced myocardium expression of ED-A 


\section{ABSTRACTS}

fibronectin, thus suppressed TGF-b1-induced fibroblast trans-differentiation and a-SMA expression, thereby leading to adverse collagen turnover, enlarged LV dilation, and deteriorated cardiac functions, similar to those from E64d-treated mice.

Conclusions E64d deteriorates LV remodelling and cardiac functions after experimental MI by affecting myofibroblast trans-differentiation via inhibition of CatS activity and suppression of fibronectin ED-A production. 